\title{
Gradual regime shifts in spatially extended ecosystems
}

\author{
Golan Bel · Aric Hagberg · Ehud Meron
}

Received: 30 August 2011 / Accepted: 7 November 2011

(C) The Author(s) 2012. This article is published with open access at Springerlink.com

\begin{abstract}
Ecosystem regime shifts are regarded as abrupt global transitions from one stable state to an alternative stable state, induced by slow environmental changes or by global disturbances. Spatially extended ecosystems, however, can also respond to local disturbances by the formation of small domains of the alternative state. Such a response can lead to gradual regime shifts involving front propagation and the coalescence of alternative-state domains. When one of the states is spatially patterned, a multitude of intermediate stable states appears, giving rise to step-like gradual shifts with extended pauses at these states. Using a minimal model, we study gradual state transitions and show that they precede abrupt transitions. We propose indicators to probe gradual regime shifts, and suggest that a combination of abrupt-shift indicators and gradual-shift
\end{abstract}

Electronic supplementary material The online version of this article (doi:10.1007/s12080-011-0149-6) contains supplementary material, which is available to authorized users.

G. Bel · E. Meron

Department of Solar Energy and Environmental Physics, BIDR, Ben-Gurion University, Sede Boqer Campus, Midreshet Ben-Gurion 84990, Israel

G. Bel

e-mail: bel@bgu.ac.il

\author{
A. Hagberg ( $\square)$ \\ Theoretical Division, Los Alamos National Laboratory, \\ Los Alamos, NM 87545, USA \\ e-mail: hagberg@lanl.gov \\ E. Meron \\ Department of Physics, Ben-Gurion University, Beer-Sheva, \\ 84105, Israel \\ e-mail: ehud@bgu.ac.il
}

indicators might be needed to unambiguously identify regime shifts. Our results are particularly relevant to desertification in drylands where transitions to bare soil take place from spotted vegetation, and the degradation process appears to involve step-like events of local vegetation mortality caused by repeated droughts.

Keywords Regime shifts • Early indicators • Alternative stable states - Pattern formation • Bistability • Front dynamics • Homoclinic snaking • Vegetation patterns $\cdot$ Desertification

\section{Introduction}

The responses of ecosystems to small environmental changes are generally divided into two categories: small, slow, and reversible, or large, abrupt, and irreversible. Various examples of the latter response have been reported, including sudden losses of transparency and vegetation in shallow lakes subject to human-induced eutrophication, coral reefs overgrown by fleshy macroalgae, and desertification induced by climate changes or human disturbances (Scheffer et al. 2001). Often the abrupt response is detrimental to the ecosystem because it causes loss of bioproductivity and biodiversity, which, in turn, affect ecosystem function and stability (Yachi and Loreau 1999; Loreau et al. 2001; Duraiappah and Naeem 2005). The heightened concern about potential ecosystem deterioration from global climate change has motivated vigorous research efforts aimed at devising early indicators of impending degradation processes (Scheffer et al. 2009).

Large sudden responses to small environmental changes have been attributed to the coexistence of two 


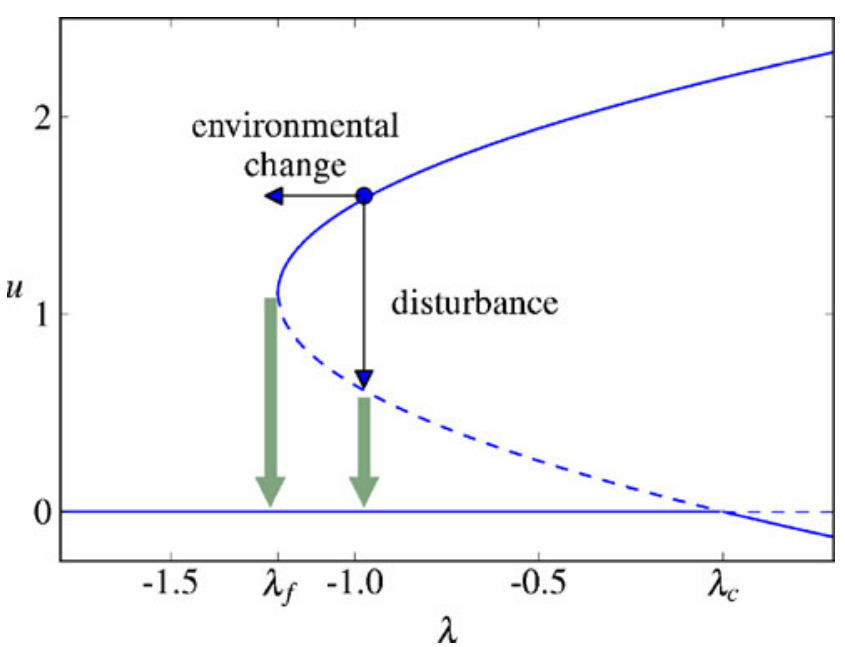

Fig. 1 Bifurcation diagram showing a bistability range of zero and nonzero $\left(u=u_{+}\right)$uniform states in Eq. 1, bounded by fold $\left(\lambda=\lambda_{\mathrm{f}}\right)$ and transcritical $(\lambda=0)$ bifurcations. Solid (dashed) lines denote stable (unstable) solutions. Illustrated are two scenarios of regime shifts, a shift induced by environmental changes (left arrow) and a shift induced by disturbances (right arrow). Parameters used to produce the diagram: $a_{2}=2.2, a_{3}=1$. The stability properties were evaluated with $d_{2}<0$ and $d_{4}=0$

alternative stable states in some range of environmental conditions (Lewontin 1969; Holling 1973; May 1977; Scheffer et al. 2001; Guttal and Jayaprakash 2007). This bistability range is bounded by instability points ${ }^{1}$ where one alternative stable state either loses stability or ceases to exist. A small environmental change that moves the system across one of these instability points can induce a transition to the other alternative stable state as the left arrow in Fig. 1 illustrates. State transitions of this kind have been termed "critical transitions", or "regime shifts."

Most studies of early indicators of regime shifts rely on a basic property of a dynamical system undergoing an instability, namely slow recovery of perturbations in the vicinity of the instability point. This property, which has been termed "critical slowing down" in analogy to a similar concept in the theory of phase transitions applies, in particular, to the instability points at the edge of a bistability range (Scheffer et al. 2001). The critical slowing down manifests itself in the increase of temporal autocorrelation and variance (Carpenter and Brock 2006; Dakos et al. 2008; Chisholm and Filotas 2009) and in the skewness of time-series distributions (Guttal and Jayaprakash 2008). Monitoring such

\footnotetext{
${ }^{1}$ The term "instability point" refers to the threshold value of a control parameter at which a system state becomes unstable, i.e., the point on the control parameter axis at which the instability occurs. This term should not be confused with the term "fixed point" which describes a stationary system state.
}

changes in empirical time series may indicate imminent regime shifts. More recent studies have considered spatially explicit models and have suggested an increase in spatial variance and correlations and peaking of spatial skewness as more feasible indicators for imminent regime shifts (Guttal and Jayaprakash 2009; Dakos et al. 2010; Donangelo et al. 2010; Dakos et al. 2011). The discrete Fourier transform in space is yet another method proposed recently for the identification of impending regime shifts (Carpenter and Brock 2010).

Two important aspects of spatially extended ecosystems have not been considered in these studies. The first is local disturbances that form confined spatial domains of the alternative stable state, even far from the instability points. The second is spatial patterning of one of the alternative stable states. These aspects are particularly relevant to desertification processes in drylands (Scheffer and Carpenter 2003). Model studies predict that transitions to the low bioproductivity, or "bare-soil," state following a precipitation downshift, never occur from a uniform vegetation state but rather from a spot-pattern state (von Hardenberg et al. 2001; Rietkerk et al. 2004). Moreover, there is a wide bistability range of the spot pattern and the bare-soil states, due to positive feedback between biomass and water (HilleRisLambers et al. 2001; Rietkerk et al. 2002) and between above-ground and below-ground biomass (Gilad et al. 2004, 2007). In this range, local vegetation disturbances, such as clear-cutting, fires, overgrazing, and infestation, often in conjunction with rainfall variability (Breshears et al. 2005), can induce the formation of bare-soil domains.

The significance of local domain formation is that it may induce front dynamics (Hagberg and Meron 1994c), i.e., dynamics of the transition zones that separate adjacent domains of the two alternative states. The dynamics may act to reduce the newly formed domains, or to extend them. The latter process suggests a third category of ecosystem response, which is neither small and slow nor large and abrupt, but rather large and slow involving gradual transitions between the two alternative stable states, in the course of which newly formed domains expand and merge (Fig. 9). This calls for the re-examination of currently suggested indicators of impending shifts and for the consideration of additional indicators.

\section{A minimal model of alternative stable states in spatially extended systems}

We study gradual state transitions using a minimal model for alternative stable states that captures an 
instability to a patterned state. The model consists of a single state variable, $u=u(x, y, t)$, which can be thought of as providing some measure for the ecosystem health (with $u \leq 0$ representing unhealthy regions). The model reads

$u_{t}=\lambda u+a_{2} u^{2}-a_{3} u^{3}-d_{2} \nabla^{2} u-d_{4} \nabla^{4} u$,

where the subscript $t$ denotes the partial time derivative, $\nabla^{2}=\partial^{2} / \partial x^{2}+\partial^{2} / \partial y^{2}$ is the Laplacian operator, and $a_{2}, a_{3}, d_{2}$, and $d_{4}$ are constant parameters. We assume that $a_{2}>0, a_{3}>0$, and $d_{4} \geq 0$. The parameter $\lambda$, which will play the role of a control parameter, represents the effect of the environment; high $\lambda$ values account for environmental amelioration that allows the growth of $u$, whereas low values (possibly negative) represent environmental stresses. The parameter $a_{2}$ represents facilitation and accounts for bistability ranges of both two uniform states and of a uniform and a patterned state, as will be explained below. The parameter $a_{3}$ accounts for processes that limit the growth of $u$, such as the depletion of a limiting resource. Finally, the space derivative terms account for short-range facilitation and long-range competition, as produced, for example, by the transport of a limiting resource toward a growing patch of some life form (Lejeune et al. 1999, 2004; Rietkerk and van de Koppel 2008; Meron 2011b). Such processes can induce pattern formation as will be clarified below. For simplicity we will assume that Eq. 1 is already in a non-dimensional form.

We wish to point out that the general conclusions to be drawn from the study of this minimal model are expected to apply to more detailed models of specific ecosystems, as they rely on universal aspects of dynamical systems such as instability phenomena. Detailed models are significant for relating the general findings to specific parameters and processes, as we explain in the "Discussion" section.

Equation 1 has a zero solution, $u=0$, representing an unhealthy state. The stability of the zero state to small perturbations can be evaluated by calculating the growth rate $\sigma(k)$ of sinusoidal perturbations (or Fourier modes) with wavenumber $k$ (or wavelength $2 \pi / k$ ). A calculation of this kind (Cross and Greenside 2009; Borgogno et al. 2009) yields the following expression:

$\sigma(k)=\lambda+d_{2} k^{2}-d_{4} k^{4}$.

Figure 2 shows graphs of $\sigma(k)$ for $d_{2}<0$ (a) and for $d_{2}>0$ (b). In both cases, there exists a threshold value $\lambda_{\mathrm{c}}$ below which any perturbation decays, implying the stability of the zero state in the range $\lambda<\lambda_{c}$. In the former case, the threshold is $\lambda_{\mathrm{c}}=0$ while in the latter it is $\lambda_{\mathrm{c}}=-d_{2}^{2} /\left(4 d_{4}\right)$. When this threshold is exceeded,

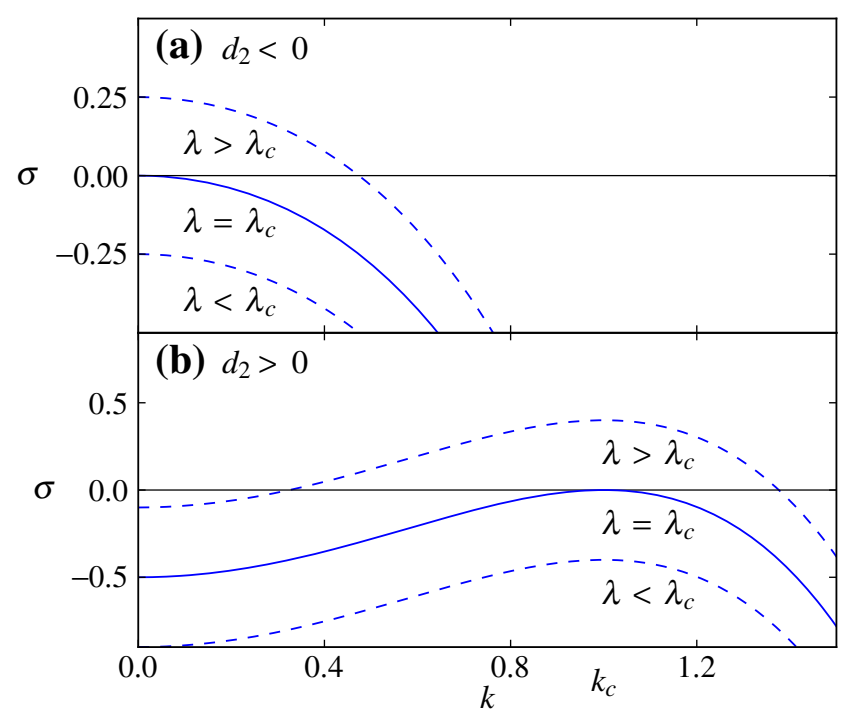

Fig. 2 Growth rates of sinusoidal perturbations with wavenumber $k$ from the zero (unhealthy) state for a $d_{2}<0$ and $\mathbf{b} d_{2}>$ 0 . When $d_{2}$ is negative, the first perturbation to grow, as $\lambda$ increases past a threshold value $\lambda_{\mathrm{c}}$, is uniform $(k=0)$. When $d_{2}$ is positive, the first perturbation to grow has a finite wavenumber $k_{\mathrm{c}}$

a critical mode $k_{\mathrm{c}}$ begins to grow. In the former case $k_{\mathrm{c}}=0$ implying that the zero solution becomes unstable to the growth of spatially uniform perturbations, while in the latter case $k_{\mathrm{c}}=\sqrt{d_{2} /\left(2 d_{4}\right)}$, implying the growth of nonuniform perturbations. This is an example of a finite-wavenumber instability that leads to a periodic pattern state. ${ }^{2}$ It is this case, $d_{2}>0$, for which the model captures short-range facilitation and long-range competition. Indeed, growth rate curves of the form shown in Fig. $2 b$ have been obtained with drylandvegetation models that specifically capture physical and ecological processes of facilitation and competition (Sherratt 2005; Gilad et al. 2007; Meron 2011b). Note the stabilizing effect of the fourth-order derivative term by reducing the growth rates of high-wavenumber modes. This effect is essential when $d_{2}>0$ but becomes insignificant when $d_{2}<0$. For simplicity, we will set $d_{4}=0$ whenever we consider the case $d_{2}<0$.

The stability range of the zero solution, $\lambda<\lambda_{\mathrm{c}}$, may include another stable solution provided $a_{2} \neq 0$. A straightforward calculation for the case $d_{2}<0$ and $a_{2}>0$ shows that in the range $\lambda_{\mathrm{f}}<\lambda<0$, where

\footnotetext{
${ }^{2}$ The term "finite-wavenumber instability" refers to an instability to the growth of a nonuniform perturbation as Fig. $2 b$ illustrates. Many other examples of finite wavenumber instabilities exist, including the Turing instability in reaction-diffusion systems and the Rayleigh-Bénard instability in thermal convection (Cross and Greenside 2009).
} 
$\lambda_{\mathrm{f}}=-a_{2}^{2} / 4$, both the zero solution and the constant solution,

$u=u_{+}=\frac{1}{2 a_{3}}\left(a_{2}+\sqrt{a_{2}^{2}+4 a_{3} \lambda}\right)$,

are stable, as the bifurcation diagram in Fig. 1 indicates. We will refer to the constant solution $u_{+}$as the "nonzero state". The bistability range is bounded from above at $\lambda=\lambda_{c}=0$, where the zero solution loses stability in a transcritical bifurcation, and from below at $\lambda=\lambda_{\mathrm{f}}=-a_{2}^{2} / 4$, where the $u=u_{+}$solution ceases to exist in a fold (saddle-node) bifurcation. For the case $d_{2}>0$, the zero state loses stability in a subcritical finite-wavenumber instability, which results in a bistability range of a uniform zero state and a periodic pattern state, $u=u_{\mathrm{p}}$, as the bifurcation diagram in Fig. 7 shows. The bistability range in this case is bounded from above at $\lambda=\lambda_{\mathrm{c}}=-d_{2}^{2} /\left(4 d_{4}\right)$, where the zero state loses stability, and from below at $\lambda=\lambda_{\mathrm{f}}$, where the periodic pattern state ceases to exist in a fold bifurcation. The threshold $\lambda_{\mathrm{f}}$ in this case is evaluated numerically.

In summary, the minimal model (Eq. 1) captures two distinct cases of regime shifts. The first applies to negative values of $d_{2}$ and involves transitions between two uniform states, $u=0$ and $u=u_{+}$, whereas the second applies to positive $d_{2}$ values and involves transitions between a uniform state, $u=0$, and a patterned state, $u=u_{\mathrm{p}}$. We will see in the next section that the second case is much more complicated because the bistability of uniform and patterned states implies the existence of many more intermediate stable states. Some of them are shown in the bifurcation diagram of Fig. 7.

\section{Pattern formation background}

Pattern formation theory provides results about front dynamics in bistable systems that are essential for understanding gradual state transitions. We describe these results below and refer the interested reader to the vast literature on this topic for more details as we proceed.

\section{Potential systems}

Equation 1 represents a potential system (Strogatz 1994). A functional ${ }^{3} V=V[u]$ can be defined with the property that it can never increase in time. We refer to

\footnotetext{
${ }^{3} \mathrm{~A}$ functional is a map that associates a number with a function, such as the value of a definite integral of some function.
}

$V$ as a potential functional ${ }^{4}$. To see how this property emerges from the model Eq. 1, consider a simpler version of this equation that does not contain the space derivative terms. This simpler form can be written as

$\frac{\mathrm{d} u}{\mathrm{~d} t}=-\frac{\mathrm{d} V}{\mathrm{~d} u}$,

where

$V=-\frac{\lambda}{2} u^{2}-\frac{a_{2}}{3} u^{3}+\frac{a_{3}}{4} u^{4}+V_{0}$,

and $V_{0}$ is an integration constant. Consider now the time derivative of $V(u)$. The function $V$ does not have an explicit time dependence, but it does depend on time implicitly through the time dependence of $u(t)$. Using the chain rule and then Eq. 4, we obtain

$\frac{\mathrm{d} V}{\mathrm{~d} t}=\frac{\mathrm{d} V}{\mathrm{~d} u} \frac{\mathrm{d} u}{\mathrm{~d} t}=-\left(\frac{\mathrm{d} u}{\mathrm{~d} t}\right)^{2} \leq 0$.

Thus, $V$ can only decrease or remain constant in time, and therefore is a potential function. Since the system tends to minimize its potential function, once it reaches a potential minimum it cannot escape from it; any sufficiently small perturbation must decay in time. Thus, minima of a potential function represent stable stationary states. The potential functional of Eq. 1 is more complicated since it contains a spatial integral over a function of $u$ and its spatial derivatives (Cross and Greenside 2009), but the general principle of dynamics that act to minimize the potential still holds ${ }^{5}$.

Bistability of uniform states

Pattern dynamics associated with the bistability of uniform states has been the subject of intense study, both in potential and non-potential systems (Fife 1988; Hagberg and Meron 1994c; Pismen 2006; Cross and Greenside 2009). The building blocks of these patterns are fronts that separate domains of different states, as Fig. $3 c$ illustrates, and much of the overall pattern dynamics can be understood in terms of the dynamics of single fronts and their mutual interactions.

In the context of Eq. 1, the bistability of uniform states amounts to $d_{2}<0$, in which case we can set $d_{4}=$

\footnotetext{
${ }^{4}$ Several alternative terms have been used in the literature to refer to potential systems and functionals. Potential systems are also called variational and gradient systems, and the functionals are also called Lyapunov and energy functionals.

${ }^{5}$ It should be noted that dynamical systems are not necessarily potential. It is often the case, however, that a system is potential in some parameter range and non-potential outside this range. Changing a parameter across the boundaries of this range is generally accompanied by the appearance of "non-potential effects", such as temporal oscillations.
} 


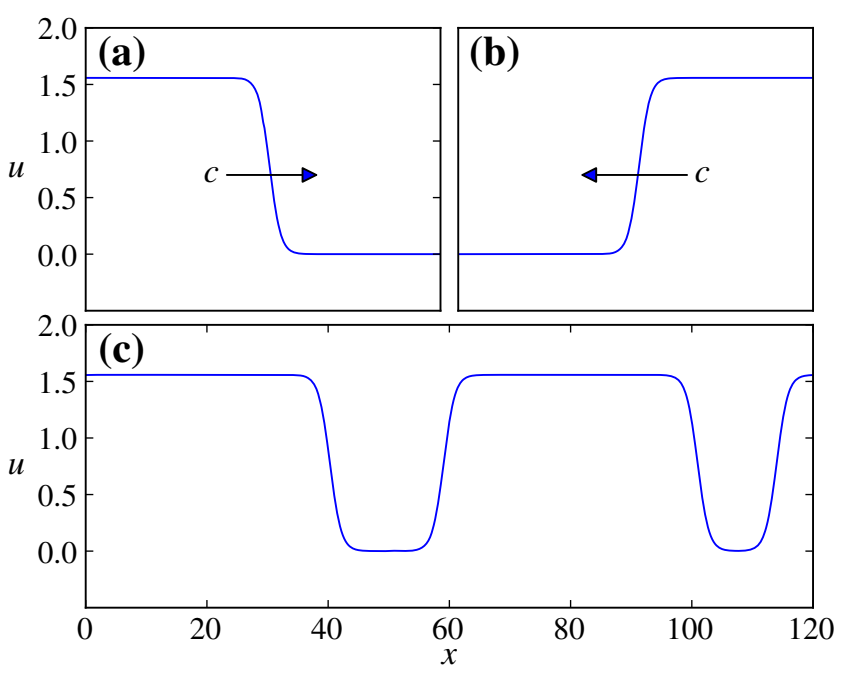

Fig. 3 Fronts as building blocks of spatial patterns for bistability of uniform states $\left(d_{2}<0\right)$. a, b A pair of symmetric front solutions of Eq. 7 representing a nonzero state propagating into the zero state for $c>0$ and vice versa for $c<0$. The arrows denote the direction of front propagation for $c>0$. $\mathbf{c}$ A pattern consisting of nonzero-state and zero-state domains separated by the two types of fronts. Parameters: $\lambda=-1.0, a_{2}=2.2, a_{3}=$ $1, d_{2}=-1, d_{4}=0$

0 (see the "A minimal model of alternative stable states in spatially extended systems" section). Equation 1 can then be written as

$u_{t}=-V^{\prime}(u)+\left|d_{2}\right| \nabla^{2} u$,

where $V$ is given by Eq. 5 and the prime denotes the derivative with respect to the argument, that is, $V^{\prime}=d V / d u$. In the following, we restrict the analysis to rectilinear fronts. For treatments that include front curvature, we refer the reader to (Hagberg and Meron 1998; Pismen 2006).

To study the dynamics of a single front, we look for a solution $u=u(\chi)$ of Eq. 7 that propagates at constant velocity $c$, where $\chi=x-c t$. Consider first a front that asymptotes to $u=u_{+}$as $\chi \rightarrow-\infty$ and to $u=0$ as $\chi \rightarrow \infty$ as Fig. 3a illustrates ${ }^{6}$. Such a solution describes a system occupied by the nonzero state far to the left and by the zero state far to the right, with a transition zone in between that moves at velocity $c$. The velocity $c$ can be found by inserting this solution form

\footnotetext{
${ }^{6} \mathrm{We}$ consider in this front analysis the infinite line $-\infty<x<\infty$, although a system of finite size $L$ must be assumed in practice and in the numerical studies to follow. The analysis on the infinite line provides good approximations for "large" finite systems, i.e., for systems much larger than the front width.
}

into Eq. 7, multiplying by $u^{\prime} \equiv d u / d \chi$ and integrating over the whole line. This gives ${ }^{7}$

$$
\begin{aligned}
c=A^{-1} \int_{-\infty}^{\infty} V^{\prime} u^{\prime} \mathrm{d} \chi & =A^{-1} \int_{u_{+}}^{0} V^{\prime} \mathrm{d} u \\
& =A^{-1}\left[V(0)-V\left(u_{+}\right)\right],
\end{aligned}
$$

where

$$
A=\int_{-\infty}^{\infty}\left(u^{\prime}\right)^{2} \mathrm{~d} \chi
$$

Along with this front solution, there exists a symmetric solution that asymptotes to $u=0$ as $\chi \rightarrow-\infty$ and to $u=u_{+}$as $\chi \rightarrow \infty$ and propagates in the opposite direction, i.e., with velocity $-c$ (see Fig. $3 b$ ).

According to Eqs. 8 and 9, the sign of the front velocity depends on the relative value of the potential $V$ at the two alternative stable states. When $V\left(u_{+}\right)<$ $V(0)$, the velocity is positive, implying that the nonzero state propagates into the zero state. Indeed, this process minimizes the potential functional of Eq. 7 as it transforms high-potential areas into low-potential areas. When $V\left(u_{+}\right)>V(0)$, the zero state propagates into the nonzero state, and when $V\left(u_{+}\right)=V(0)$, the front is stationary $(c=0)$. Note that the same conclusions also hold for the symmetric front; for $V\left(u_{+}\right)<V(0)$, the nonzero state propagates into the zero state and vice versa for $V\left(u_{+}\right)>V(0)$. The potential values of the two states are affected by the control parameter $\lambda$ (see Eq. 5). The particular value, $\lambda=\lambda_{\mathrm{m}}$, for which the potential values of the two states are equal, is called the Maxwell point (see, for example, Pismen 2006). For $\lambda>\lambda_{\mathrm{m}}$, the nonzero state propagates into the zero state, and for $\lambda<\lambda_{\mathrm{m}}$, the zero state propagates into the nonzero state. A straightforward calculation gives $\lambda_{\mathrm{m}}=-2 a_{2}^{2} /\left(9 a_{3}\right)$. More generally, we will define the Maxwell point as the parameter value at which $c=0$. This definition will be useful for more complex models of bistable systems, the potential functionals of which are not known.

Consider now a domain of the zero state in a background of the nonzero state within the bistability range $\lambda_{\mathrm{f}}<\lambda<\lambda_{\mathrm{c}}$. Above the Maxwell point $\left(\lambda>\lambda_{\mathrm{m}}\right)$, such a domain will shrink in size and the two fronts that enclose the domain will get closer to one another. It is during this stage that front interactions become significant. For the model Eq. 1, these interactions are attractive, and the dynamics culminate in the collapse of the zero-state domain and the convergence to a uniform nonzero state as Fig. 4a demonstrates. At the

\footnotetext{
${ }^{7}$ In deriving Eq. 8, we use integration by parts and the fact that $u^{\prime} \rightarrow 0$ as $\chi \rightarrow \pm \infty$.
} 

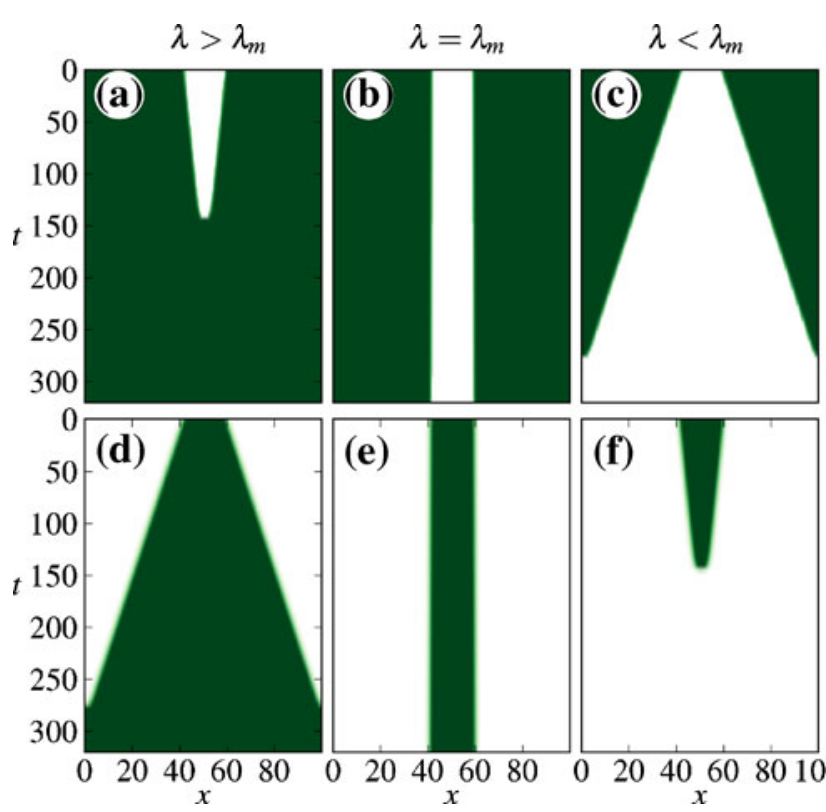

(f)

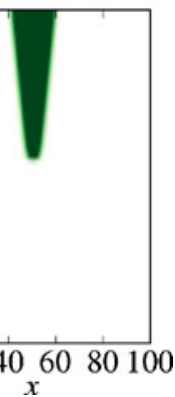

Fig. 4 Space-time plots showing the dynamics of initial zerostate domains a-c and nonzero-state domains $\mathbf{d}-\mathbf{f}$ for bistability of uniform states $\left(d_{2}<0\right)$. Dark shades represent the healthy nonzero state. To the right of the Maxwell point $\left(\lambda>\lambda_{\mathrm{m}}\right)$, an initially small zero-state domain shrinks a, while an initially small nonzero-state domain expands d. Both initial conditions culminate in the uniform nonzero state. To the left of the Maxwell point $\left(\lambda<\lambda_{\mathrm{m}}\right)$, an initially small zero-state domain expands $\mathbf{c}$, while an initially small nonzero-state domain shrinks f. In that case, both initial conditions culminate in the uniform zero state. At the Maxwell point, the initial domains are stationary, provided they are not too small for the attractive front interactions to be significant b, e. Parameters: $a_{2}=2.2, a_{3}=1, d_{2}=-1, d_{4}=0$, $\mathbf{a}, \mathbf{d} \lambda=-1.06, \mathbf{b}, \mathbf{e} \lambda=\lambda_{\mathrm{m}}=-1.076, \mathbf{c}, \mathbf{f} \lambda=-1.12$

Maxwell point, that is, at exactly $\lambda=\lambda_{\mathrm{m}}$, the zero-state domain will remain of constant size (Fig. 4b), while below the Maxwell point $\left(\lambda<\lambda_{\mathrm{m}}\right)$, it will grow in size until it occupies the whole system (Fig. 4c). A domain of the nonzero state in a background of the zero state will expand above the Maxwell point, remain of fixed size at the Maxwell point, and shrink below it as Fig. 4d-f shows.

\section{Bistability of uniform and patterned states}

The main difference between the bistability of uniform and patterned states, and the bistability of two uniform states is that the former generally implies a multistability of states, at least in a subrange of the bistability range. As we will see below, this subrange may involve many stable patterned states, infinitely many in an infinite system, that can be viewed as spatial mixtures of the periodic pattern state and the uniform zero state.

As in the case of two stable uniform states, much insight about the structure and dynamics of spatial pat-

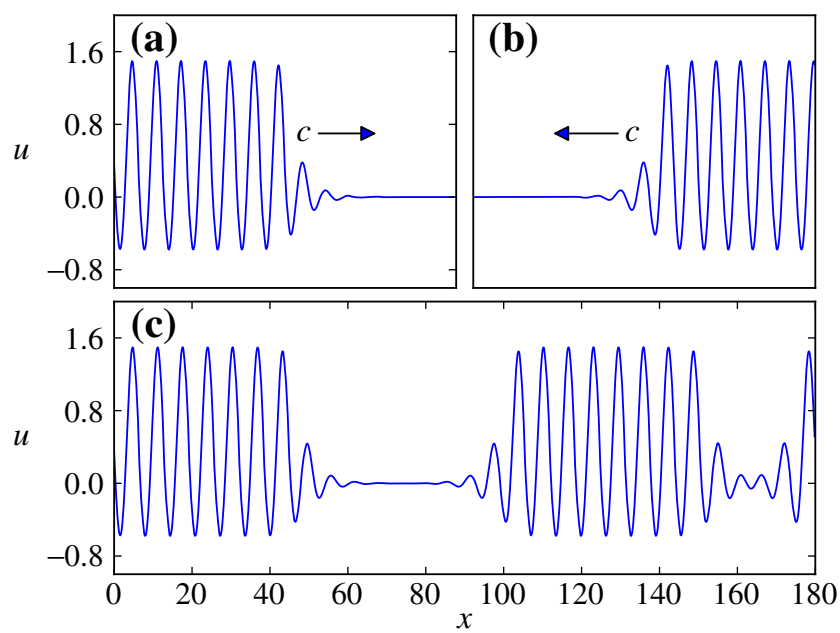

Fig. 5 Fronts as building blocks of spatial patterns for bistability of uniform and patterned states $\left(d_{2}>0\right)$. a, b A pair of symmetric front solutions of Eq. 1 representing a patterned state propagating into the zero state for $c>0$ and vice versa for $c<0$. Unlike the case of two uniform states, there exists a control parameter range, rather than a single value, in which the two fronts are stationary $(c=0)$. $\mathbf{c}$ A pattern consisting of patternedstate and zero-state domains separated by the two types of fronts. Parameters: $\lambda=-1.28, a_{2}=1.82, a_{3}=1, d_{2}=2, d_{4}=1$

terns can be obtained from the dynamics of single fronts and their interactions. The fronts separate domains of uniform and patterned states as shown in Fig. 5. Such fronts, however, behave differently in one important respect compared with fronts that separate two uniform states - they are stationary in a range of the control parameter, $\lambda_{1}<\lambda<\lambda_{2}$, that includes the Maxwell point, rather than being stationary at the Maxwell point only (Pomeau 1986).

To study the implications of this front property, we consider Eq. 1 with $d_{2}>0^{8}$. Figure 6 a-c shows numerical solutions of Eq. 1 describing the dynamics of a zero-state domain in a background of a periodic pattern within the range of bistability, $\lambda_{\mathrm{f}}<\lambda<\lambda_{\mathrm{c}}$. This range can be divided into three parts where different behaviors take place: (a) $\lambda_{2}<\lambda<\lambda_{\mathrm{c}}$ where the zerostate domain diminishes in size and disappears, (b) $\lambda_{1}<\lambda<\lambda_{2}$ where the zero-state domain retains a fixed size because the fronts are stationary, and (c) $\lambda_{\mathrm{f}}<$ $\lambda<\lambda_{1}$ where the zero-state domain expands until it occupies the whole system. In the first range, the system

\footnotetext{
${ }^{8}$ Note that with $d_{2}>0$, Eq. 1 is equivalent, with appropriate rescaling of $u$ and the space coordinates, to the SwiftHohenberg equation, $u_{t}=r u+\alpha u^{2}-u^{3}-\left(\nabla^{2}+k_{0}^{2}\right)^{2} u$ (Croos and Greenside 2009).
} 


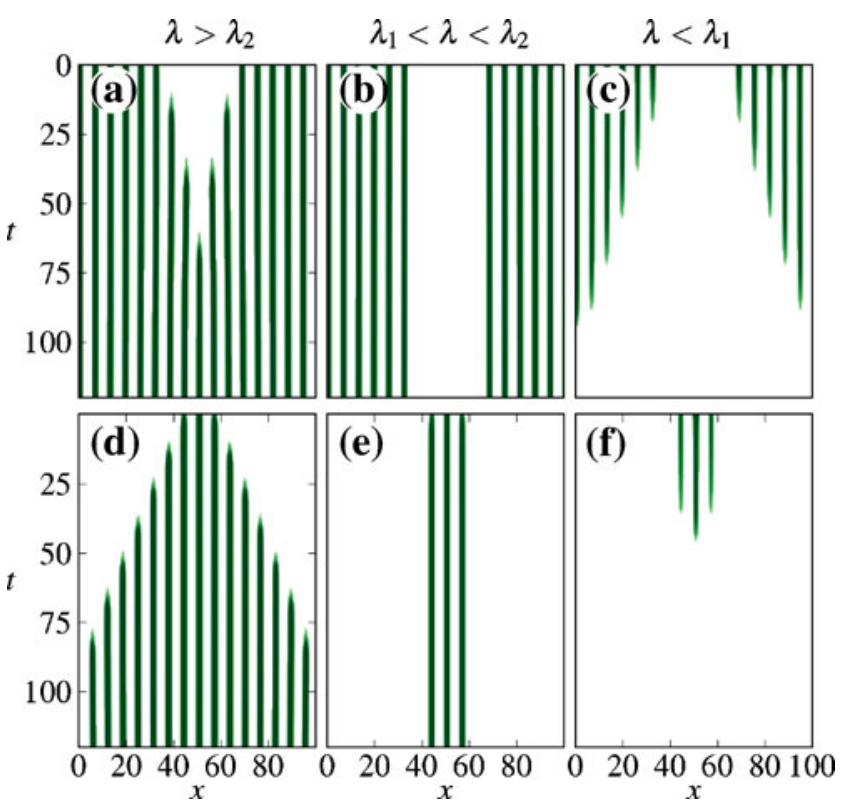

Fig. 6 Space-time plots showing the dynamics of initial zerostate domains a-c and pattern-state domains d-f for bistability of uniform and patterned states $\left(d_{2}>0\right)$. Unlike the case of two uniform states shown in Fig. 4, a control parameter range exists where the initial zero and pattern-state domains quickly converge to stationary hole solutions $\mathbf{b}$ and localized solutions $\mathbf{e}$, respectively. The dynamics resemble that of two uniform states only outside this control parameter range as a, $\mathbf{c}$ and $\mathbf{d}, \mathbf{f}$ show. Parameters: $a_{2}=1.82, a_{3}=1, d_{2}=2, d_{4}=1, \mathbf{a} \lambda=-1.22, \mathbf{b} \lambda=$ -1.28 , c $\lambda=-1.375$, d $\lambda=-1.18$, e $\lambda=-1.28, \mathbf{f} \lambda=-1.355$, $\lambda_{1}=-1.353$, and $\lambda_{2}=-1.266$

converges to the stable periodic pattern state. In the third range, it converges to the stable uniform zero state. In the range in between, it converges to a stable hole solution, that is, a solution describing a zero-state domain of fixed size in an otherwise periodic pattern. For a reason to become apparent shortly, the range $\lambda_{1}<\lambda<\lambda_{2}$ is called the snaking range. Analogous behavior is found for an initial pattern-state domain in a background of the zero state as Fig. 6d-f shows. The pattern-state domain of fixed size in the snaking range (Fig. 6e) describes a stable localized solution.

The stable hole solution the system converges to in Fig. $6 \mathrm{~b}$ is just one example out of many other stable hole solutions that exist in the range $\lambda_{1}<\lambda<\lambda_{2}$. The bifurcation diagram in Fig. 7 shows one family of such solutions. The family emerges from the periodic pattern branch near the fold bifurcation at $\lambda=\lambda_{\mathrm{f}}$, snakes down, and terminates at the unstable periodic pattern branch near the zero state. The upper branches of this solution family describe small holes in an otherwise periodic pattern that widen in the descent toward the zero state. The full bifurcation diagram of solutions is

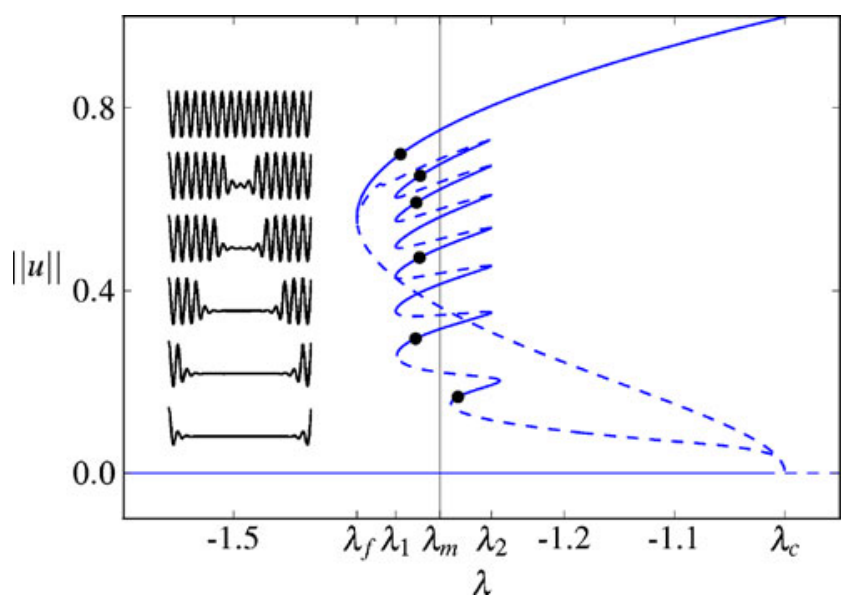

Fig. 7 Bistability of a uniform zero state and a periodic pattern state $\left(u=u_{\mathrm{p}}\right)$ in Eq. 1 . The vertical axis, $\|u\|$, represents the $L 2$ norm of $u$, a global measure involving the square root of the spatial integral of $u^{2}$. Specifically, $\|u\|$ satisfies $\|u\|^{2}=$ $L^{-1} \int_{0}^{L} u^{2} \mathrm{~d} x$, where $L$ is the system size. Solid (dashed) lines denote stable (unstable) solutions. The bistability range, $\lambda_{\mathrm{f}}<$ $\lambda<\lambda_{\mathrm{c}}$, includes a snaking range, $\lambda_{1}<\lambda<\lambda_{2}$, in which families of localized and hole solutions exist. Shown is a family of hole solutions that bifurcates from the periodic pattern solution and snakes down, approaching the zero solution. The branches of this family describe holes of increasing sizes as the insets on the left show. The insets correspond to the solid circles on the stable solution branches. The vertical line denotes the Maxwell point $\lambda=\lambda_{\mathrm{m}}$. Parameters: $a_{2}=1.82, a_{3}=1, d_{2}=2, d_{4}=1$. For these parameters, $\lambda_{\mathrm{m}}=-1.31, \lambda_{1}=-1.353$, and $\lambda_{2}=-1.266$

more involved and contains additional periodic pattern branches and many more states that are intermediate between the periodic and the zero states. We refer the reader to (Kozyreff and Chapman 2006; Burke and Knobloch 2007; Knobloch 2008; Bergeon et al. 2008; Chapman and Kozyreff 2009) for additional information and for fuller descriptions of the bifurcation structure.

The solution structure described above for one space dimension extends to two space dimensions with richer solution forms (Lloyd et al. 2008; Avitabile et al. 2010; McCalla and Sandstede 2010). We illustrate some of these solutions in Fig. 8. The leftmost and rightmost panels show the alternative stable states, periodic hexagonal pattern (left) and zero (right). The panels in between show intermediate states consisting of hole structures of different sizes, combinations of hole and localized structures, and localized structures of different size. Intermediate states of this kind have been found in many other models with bistability ranges of uniform and patterned states, including models of dryland vegetation (Meron et al. 2007; Gilad et al. 2007; Tlidi et al. 2008). 


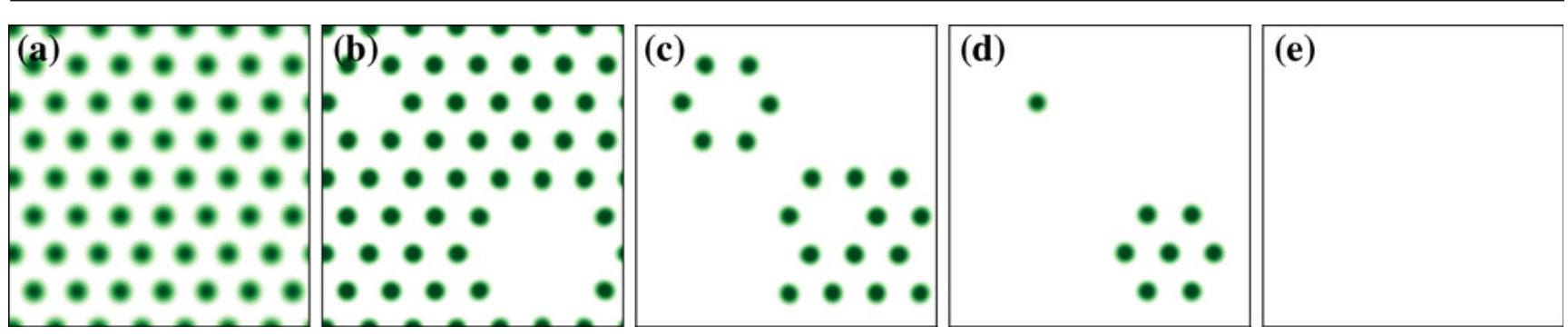

Fig. 8 Examples of 2D patterns in the bistability range of a uniform state (e) and a periodic pattern state (a) in Eq. (1). The examples include a spatial mixture of hole structures of different sizes (b), a spatial mixture of localized structures of different sizes (d), and a combination of localized and hole structures (c). Dark shading indicates high $u$ values. Parameters: $\lambda=-1.7, a_{2}=$ $2.2, a_{3}=1, d_{2}=2, d_{4}=1, x=y=[0,50]$

\section{Gradual regime shifts}

Two major scenarios of state transitions in spatially extended ecosystems can be conceived: (a) changes of a control parameter representing an environmental factor, such as precipitation, across an instability point and (b) disturbances of state variables, e.g., biomass removal, that move the system outside the state's basin of attraction. The former scenario describes an abrupt global transition occurring across the whole system (left arrow in Fig. 1), but may not be realizable if the system state is not sufficiently close to the instability point. The latter scenario may also be regarded as an abrupt global transition if the disturbance itself is global, i.e., occurring across the whole system (right arrow in Fig. 1). While global disturbances do occur, more likely are disturbances of smaller spatial extent that are strong enough to induce local state transitions, that is, the formation of alternative state domains. The expansion and coalescence of these domains result in gradual global transitions.

\section{Transitions between uniform states}

The results of the "Bistability of uniform states" section (Fig. 4) suggest two possible responses of the uniform nonzero state $u=u_{+}$to confined strong disturbances, depending on the control parameter value relative to the Maxwell point: (a) a complete recovery to the uniform nonzero state when $\lambda>\lambda_{\mathrm{m}}$ and (b) a gradual global transition to the zero state when $\lambda<\lambda_{\mathrm{m}}$. The second response is shown in panel (a) of Fig. 9. It involves the expansion and coalescence of zerostate domains at a rate determined by the distance to the Maxwell point; the closer $\lambda$ to $\lambda_{\mathrm{m}}$, the slower the gradual global transition. As the downward arrow in panel (c) indicates, such a gradual transition can
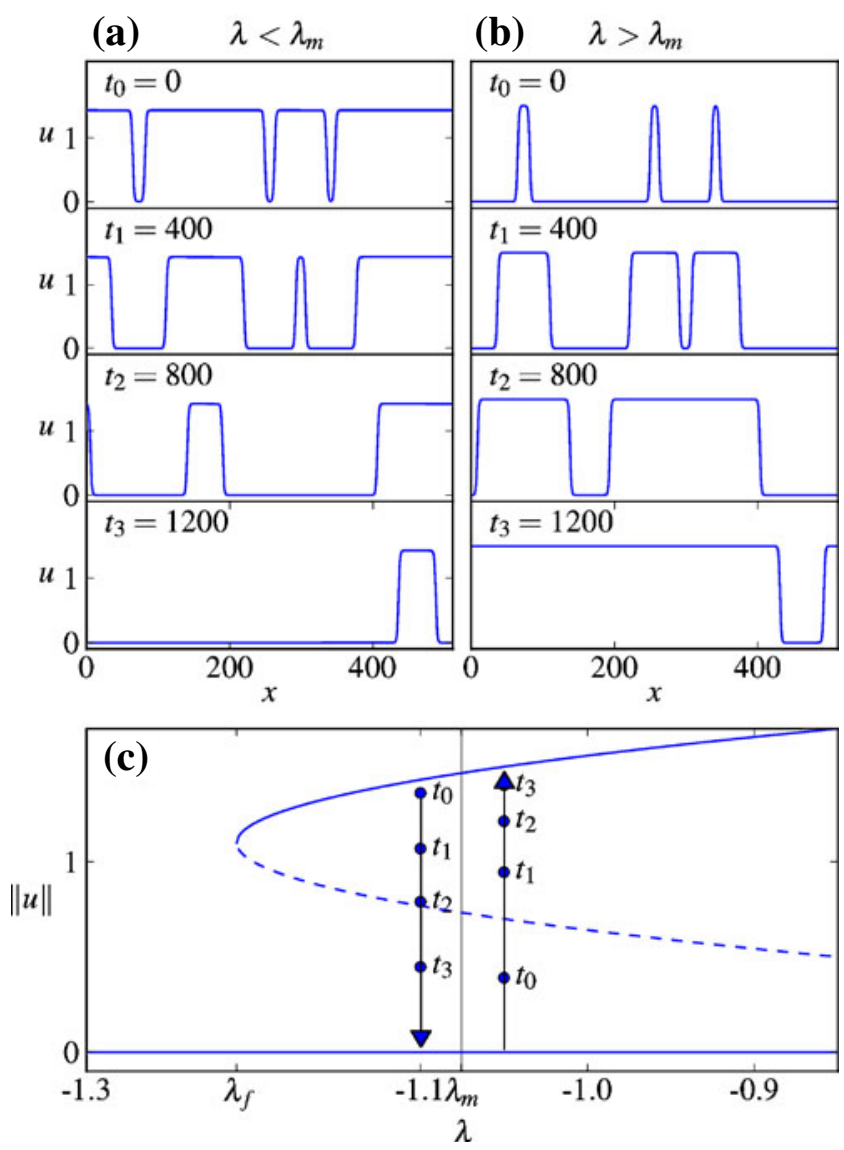

Fig. 9 Gradual state transitions for bistability of two uniform states. To the left of the Maxwell point, $\lambda<\lambda_{\mathrm{m}}$, a disturbed nonzero state gradually degrades toward the zero state, as the snapshots in a show. To the right of the Maxwell point, $\lambda>\lambda_{\mathrm{m}}$, a near-zero degraded state gradually recovers and approaches the nonzero state, as the snapshots in $\mathbf{b}$ show. The gradual degradation can occur at $\lambda$ values significantly higher than $\lambda_{\mathrm{f}}$, the threshold of abrupt degradation, as the downward arrow in c shows. Likewise, the gradual recovery can occur at $\lambda$ values significantly lower than $\lambda=0$, the threshold of abrupt recovery, as the upward arrow shows. The solid circles on the arrows denote the values of $\|u\|$ at the times the snapshots in $\mathbf{a}$ and $\mathbf{b}$ were taken. Parameters: $a_{2}=2.2, a_{3}=1, d_{2}=-1, d_{4}=0, \mathbf{a} \lambda=-1.1$, b $\lambda=-1.05$. For these parameters, $\lambda_{\mathrm{m}}=-1.076$ 
occur far from the fold bifurcation of the nonzero state at $\lambda=\lambda_{\mathrm{f}}$, and thus well before early indicators based on the proximity to the fold bifurcation become effective.

Likewise, efforts to recover the uniform nonzero state from the zero state by creating nonzero-state domains can follow two scenarios: (a) decay of the nonzero-state domains and the convergence back to the zero state when $\lambda<\lambda_{\mathrm{m}}$, and (b) expansion and coalescence of nonzero-state domains, until a global recovery of the nonzero state is attained, when $\lambda>\lambda_{\mathrm{m}}$. The second scenario is shown in panel (b) of Fig. 9. As the upward arrow in panel (c) indicates, such a gradual global recovery can occur well before the abrupt transition to the nonzero state at $\lambda=0$.

\section{Transitions between uniform and patterned states}

In this case, the healthy state $\left(u=u_{\mathrm{p}}\right)$ is spatially patterned, and the response to confined disturbances is more involved. Outside the snaking range, the behavior is similar to the case of two uniform states discussed above. For $\lambda>\lambda_{2}>\lambda_{\mathrm{m}}$, domains of the zero state shrink and disappear and the patterned state completely recovers. For $\lambda<\lambda_{1}<\lambda_{\mathrm{m}}$, such domains expand, merge, and induce a gradual global transition to the zero state. Inside the snaking range, $\lambda_{1}<\lambda<\lambda_{2}$, the impact of confined disturbances remains local as the fronts that bound a newly formed zero-state domain are stationary (see Fig. 6b, e). That is, a local disturbance can only induce a transition to a stable hole structureone of the many stable intermediate states within the snaking range (see Fig. 7).

In reality, ecosystems are subjected both to disturbances and to environmental variability. The combination of the two can lead to gradual global transitions even within the snaking range, as we now demonstrate. Consider the response of a hole solution, corresponding to an intermediate state up the snaking range, to a time-periodic modulation of the control parameter, $\lambda=$ $\lambda_{0}(1+\varepsilon \cos (\omega t))$. In the context of dryland vegetation, for example, the hole solution may represent a vegetation pattern with locally removed biomass patches, while the modulated control parameter may represent a series of droughts. The two parameters $\lambda_{0}$ and $\varepsilon$ are chosen such that $\lambda_{0}(1-\varepsilon)<\lambda_{1}$ and $\lambda_{0}(1+\varepsilon)<\lambda_{2}$, where $\lambda_{1}<\lambda_{0}<\lambda_{2}$. This choice amounts to a periodic escape of the control parameter out of the left side of the snaking range. A possible response of the system is shown in Fig. 10a. The initial domain of the zero state invades the adjacent domains of the pattern state by eliminating a pair of edge humps. This process oc-

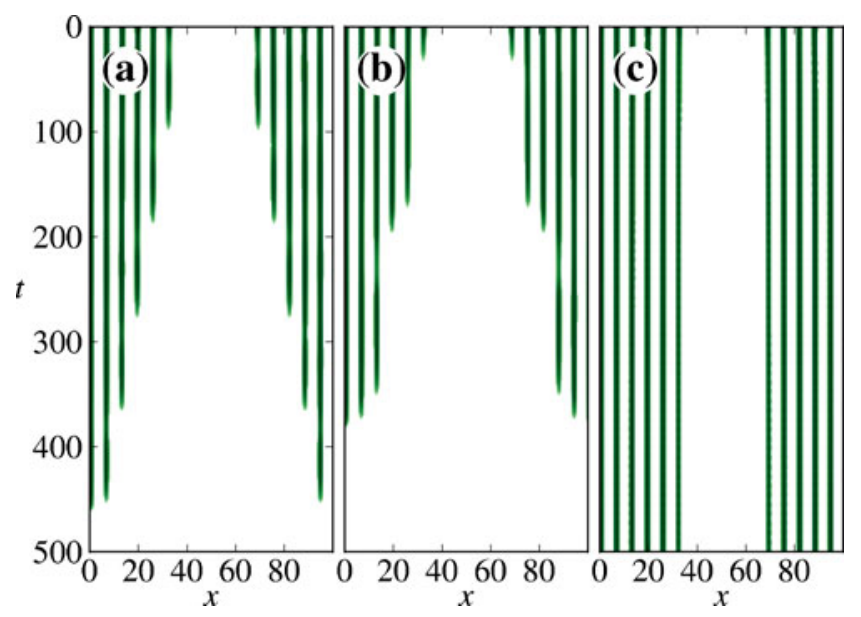

Fig. 10 Space-time plots showing gradual state transitions to the zero state for bistability of uniform and patterned states, within the snaking range under time-periodic modulation of the control parameter. When the modulation time scale is comparable to the transition time between adjacent hole solutions, the transition involves hopping from one hole solution to the next at each modulation cycle $(a)$. When the modulation time scale is sufficiently longer than the transition time, the hopping is to every second hole solution at each cycle $(b)$. When the modulation time scale is sufficiently shorter than the transition time, no transition is taking place $(c)$. Parameters: $\lambda_{0}=-1.35, a_{2}=1.82, a_{3}=1, d_{2}=2, d_{4}=$ $1, \varepsilon=0.013, \mathbf{a} \omega=0.07, \mathbf{b} \omega=0.007, \mathbf{c} \omega=0.7$

curs every cycle of the periodic modulation, during the time interval outside the snaking range. The result is a step-like dynamics down the snaking branches with extended pauses in each branch that is visited. For this scenario to happen, the time scale of the periodic modulation should be of the same order of magnitude as the state transition time scale, or $\omega \sim \sqrt{\lambda_{1}-\lambda_{\min }}$, where $\lambda_{\min }=\lambda_{0}(1-\varepsilon)$ (Burke and Knobloch 2006). If the modulation is too slow, the dynamics can skip one or more intermediate states in the descent toward the zero state (Fig. 10b), and if it is too fast, the zero domain remains confined and no global transition takes place (Fig. 10c).

To demonstrate the difference between abrupt and gradual state transitions, we numerically solved Eq. 1 in two space dimensions, once for $\lambda<\lambda_{\mathrm{f}}$ and once for $\lambda_{\mathrm{f}}<$ $\lambda<\lambda_{1}$, starting from the same initial state. Animations of these solutions are shown in Online Resource 1 and Online Resource 2. In the former case, an abrupt transition takes place in which the system globally collapses to the zero state on a short time scale. In the latter case, a gradual transition takes place in which zero-state domains expand and merge until a uniform zero state is reached. The transition is global but occurs on a much longer time scale. 


\section{Indicators of gradual regime shifts}

Gradual global transitions involve significant changes in global measures of $u$, such as the spatial average $u_{\text {ave }}$, or the area fraction, $u_{\mathrm{cov}}$, covered by patches of $u>0$. A transition down to the zero state involves the expansion of zero-state domains, and thus a decrease of $u_{\text {ave }}$ and $u_{\text {cov }}$, while a transition to the healthy state involves an increase of these quantities. Gradual transitions are also characterized by changes in spatial correlations. The dynamics down to the zero state involve a decrease of spatial correlations, for the nonzero-state domains become more and more isolated from one another, while the dynamics up to the healthy state involve an increase of spatial correlations. To quantify changes of spatial correlations, we define a correlation length, $\Lambda$, as

$$
\begin{array}{r}
\Lambda(t)=\frac{1}{L} \int_{0}^{L}|C(x, t)| x \mathrm{~d} x, \\
C(x, t) \equiv \frac{\int_{0}^{L-x} u\left(x^{\prime}, t\right) u\left(x^{\prime}+x, t\right) \mathrm{d} x^{\prime}}{\int_{0}^{L} u_{\mathrm{p}}(x) u_{\mathrm{p}}(x) \mathrm{d} x},
\end{array}
$$

where $u_{\mathrm{p}}$ is the stationary periodic solution at the same parameter values of $u$, representing the original undisturbed state.

Figures 11 and 12 show graphs of $u_{\text {ave }}, u_{\text {cov }}$, and $\Lambda$ vs. time for bistability of uniform and patterned states and time-periodic modulation of the control parameter that induces gradual state transitions. When the escape from

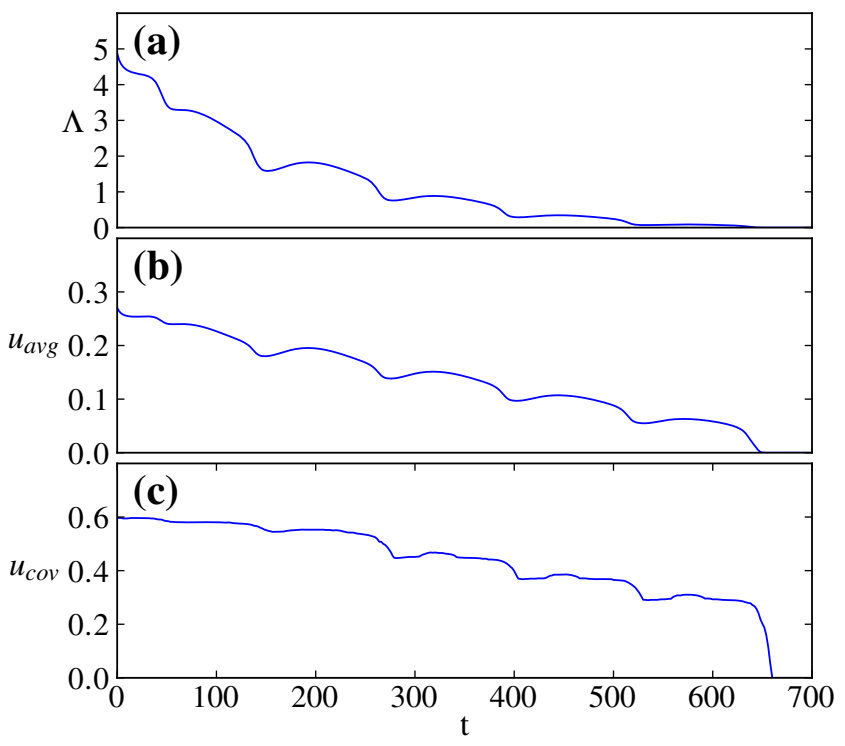

Fig. 11 Temporal changes of the correlation length $\Lambda$, the spatial average $u_{\text {ave }}$, and the coverage $u_{\text {cov }}$ as indicators of gradual degradation. Parameters: $\lambda=-1.3475, a_{2}=1.82, d_{2}=$ $2, d_{4}=1, \varepsilon=0.01, \omega=0.05$
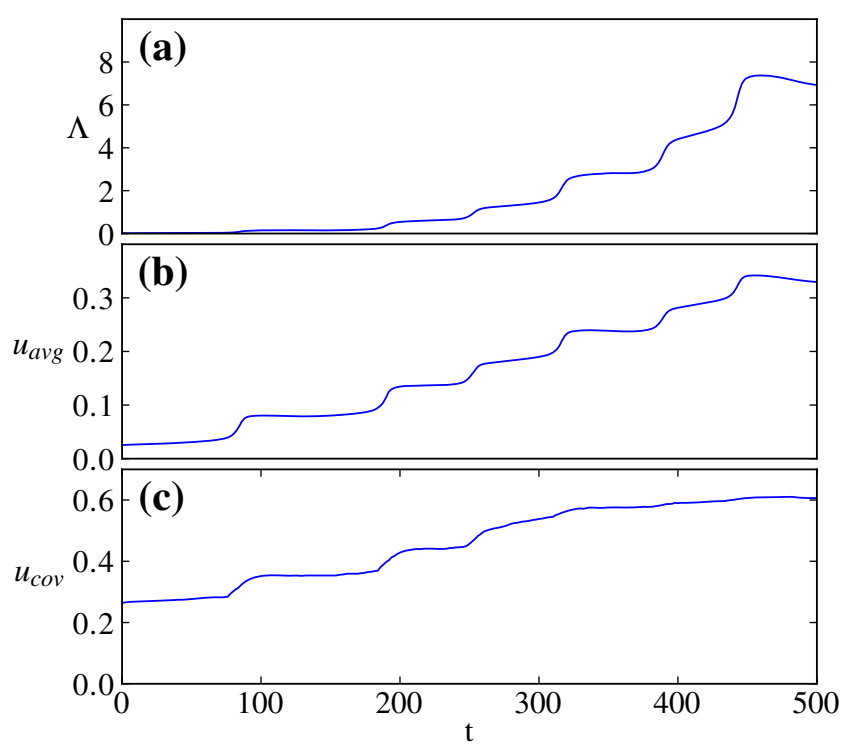

Fig. 12 Temporal changes of the correlation length $\Lambda$, the spatial average $u_{\text {ave }}$, and the coverage $u_{\text {cov }}$ as indicators of gradual recovery. Parameters: $\lambda=-1.26, a_{2}=1.82, d_{2}=2, d_{4}=1, \varepsilon=$ $0.0127, \omega=0.05$

the snaking range is to the left of the Maxwell point, these quantities decrease in time, indicating a gradual degradation to the uniform zero state (Fig. 11). When the escape is to the right of this point, they increase in time, indicating a gradual recovery of the healthy patterned state (Fig. 12). Monitoring temporal changes of these quantities can give indications whether the system is in the course of a gradual state transition. Although these indicators provide information about state transitions in process, rather than warning signals of impending transitions, they can still function as early warning signals, since the slow gradual nature of the transitions leaves time for response or for preventive measures to be taken.

\section{Discussion}

Bistable ecosystems are naturally subjected to local disturbances of various kinds, some of which may be strong enough to form small spatial domains of the alternative stable state. The dynamics of such domains can lead to gradual rather than abrupt regime shifts, as discussed in the "Gradual regime shifts" section. Gradual regime shifts are affected by the types of the alternative stable states. When the two states are spatially uniform, the shifts are determined by the value of the control parameter relative to the Maxwell point. Values smaller than the Maxwell point induce gradual degradation to the zero state, while larger values in- 
duce gradual recovery to the uniform nonzero state. When one of the alternative stable states is spatially patterned, the system becomes multi-stable; a variety of stable intermediate states (Fig. 8) appear in the snaking range (Fig. 7), which includes the Maxwell point. Gradual regime shifts occur only outside the snaking range, but time-periodic variations of the control parameter can drive the system out of this range and induce gradual regime shifts with extended pauses in consecutive intermediate states (Fig. 10).

These results shed new light on current efforts to devise effective early indicators for impending regime shifts. The results first suggest that indicators that are based on the proximity to an instability point are not effective in identifying gradual shifts, since such shifts can take place well before the instability point is reached. This conclusion particularly holds for the case of two uniform stable states where gradual state transitions can take place as far from the fold bifurcation $(\lambda=$ $\lambda_{\mathrm{f}}$ ) as the vicinity of the Maxwell point (see down arrow in Fig. 9c). In the case of bistability of uniform and patterned states, the use of such indicators may not be conclusive because of the multitude of fold bifurcations associated with the snaking structure (see Fig. 7). An approach to a fold bifurcation near $\lambda_{2}$ may be accompanied by increasing temporal and spatial correlations, very much like the approach to the fold bifurcation at $\lambda_{\mathrm{f}}$, but would imply an impending gradual recovery rather than abrupt degradation. Likewise, increasing spatial correlations may indicate a gradual recovery in progress (see Fig. 12a) rather than impending abrupt degradation.

These considerations suggest that a single indicator may not be sufficient for identifying regime shifts and that a combination of two types may be needed: indicators sensitive to abrupt shifts and indicators sensitive to gradual shifts. Applying spatial correlations as a single indicator (Dakos et al. 2010), for example, may not distinguish an impending abrupt degradation from a gradual recovery process, but measuring additionally the spatial average or coverage of a state variable can resolve this ambiguity: No significant change in these quantities would imply an impending abrupt degradation $^{9}$, whereas a significant increase would indicate a gradual recovery process. Applying gradual-shift indicators alone may not be conclusive either if the system state is not sufficiently disturbed to create domains of the alternative state; the indicators may rule out

\footnotetext{
${ }^{9}$ This is because the increase in the spatial correlations as an instability point is approached occurs within a narrow range of the control parameter over which the system's state hardly changes.
}

gradual transitions but would not predict an impending abrupt shift. A deeper analysis of indicator combinations can be pursued for specific contexts, using detailed models or actual field data.

We may expect the response of an ecosystem to environmental changes and disturbances to be sensitive to the snaking structure of intermediate states. The model Eq. 1 has a rather simple snaking structure in which all intermediate states are aligned to $\lambda=\lambda_{1}$ on the left and to $\lambda=\lambda_{2}$ on the right. However, additional snaking structures have been studied (Firth et al. 2007; Dawes 2008), and many more can be envisaged; a few of them are schematically illustrated in Fig. 13. The slanted alignments of the intermediate states bear on the degree to which state transitions are gradual. The degradation to the unhealthy zero state is more gradual in Fig. 13a and more abrupt in Fig. 13b, compared with the case of vertical alignment. Likewise, the recovery of the healthy state is more gradual in Fig. 13c and more abrupt in Fig. 13d. The manner by which the snaking structure is affected by various biotic and abiotic processes in specific ecosystems is an open problem that, to the best of our knowledge, has not been addressed yet.

Water-limited vegetation provides an intriguing application of the results reported here. Model studies indicate that increased aridity can result in bistability of a vegetation pattern state and a uniform bare-soil state (von Hardenberg et al. 2001; Rietkerk et al. 2004). The results of the "Gradual regime shifts" section then suggest that variable environmental stresses, such as a series of droughts, can gradually drive a global

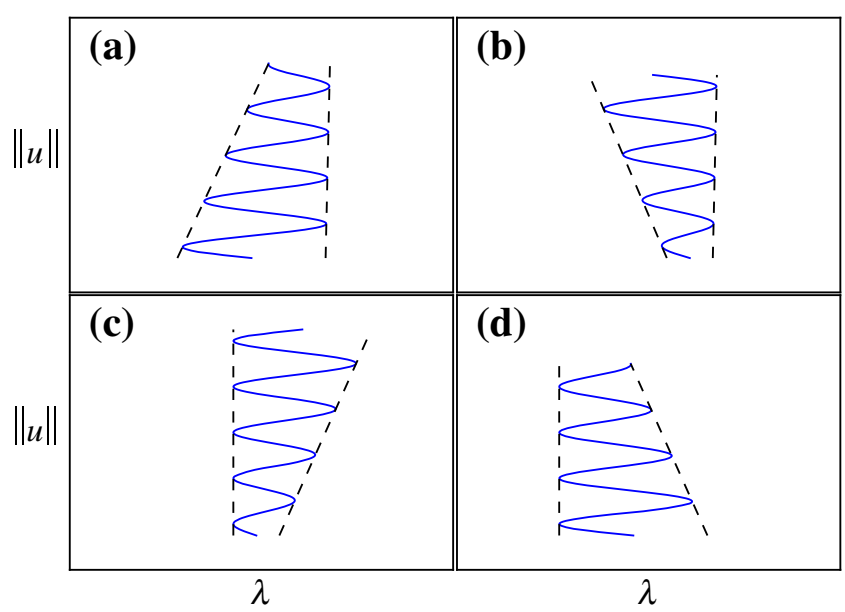

Fig. 13 Schematic illustration of the expected effect of the snaking structure on gradual degradation and recovery processes. Compared to the snaking structure in Fig. 7, a represents more gradual degradation, b more abrupt degradation, c more gradual recovery, and $\mathbf{d}$ more abrupt recovery 
transition to the bare-soil state. The global transition consists of a succession of local transitions which take the system from one intermediate state to another with a gradual loss of bioproductivity. The local transitions are characterized by step-like expansions of bare-soil domains involving the mortality of vegetation edge spots. Step-like transitions of this kind have been found recently in a cellular-automaton type vegetation model (Bailey 2010) and are consistent with field observations (Mueller et al. 2005). Gradual desertification of this kind may not be easily noticeable because of the long time intervals the system spends in the intermediate stable states and the local nature of the transitions among these states. It can be identified, however, by evaluating the correlation length and the fraction of area covered by vegetation, using remote sensing data and following them in time.

We restricted our study to the case where the patterned state represents a periodic pattern, but this may not always be the case. In the context of dryland vegetation, global competition for the limited water resource allows for scale-free patterns, characterized by powerlaw patch-size distributions (Scanlon et al. 2007; Kéfi et al. 2007; Manor and Shnerb 2008; von Hardenberg et al. 2010). The extent to which the concept of gradual state transitions applies to this case requires further studies. However, when conditions of global competition develop (von Hardenberg et al. 2010), increasing stress results in the appearance of a characteristic length, an observation that by itself can be used to identify impending degradation (Kéfi et al. 2011).

The study presented here is based on a minimal model that captures universal aspects of spatially extended bistable systems, such as gradual state transitions involving front propagation, and the snaking structure of intermediate states. These universal aspects are expected to appear in more detailed ecosystem models as well (Borgogno et al. 2009; Meron 2011a). Extending this study to such models can provide information about the dependence of the front velocity and of the snaking structure on specific ecological and physical parameters, such as various plant traits, soil properties, topography, and environmental stresses. This information, in turn, can shed light on the nature of desertification and vegetation recovery processes. For example, factors affecting facilitation, such as increased runoff interception in vegetation patches, may lead to slanted snaking, as shown in Fig. 13d, and increase the abruptness of the recovery process.

There are additional universal aspects of the bistability of uniform states that are not captured by the minimal model Eq. 1. These include repulsive front interactions (Hagberg and Meron 1994c) and global constraints (Meerson and Sasorov 1996; Kletter et al. 2012) that prevent the convergence to a uniform state, and non-potential effects such as traveling waves and spatiotemporal chaos (Hagberg and Meron 1994b, a). These aspects can be studied using two-variable models such as the FitzHugh-Nagumo model (see, e.g., Hagberg and Meron 1994c). Another direction that calls for further exploration is stochastic spatially extended models with non-smooth potentials (Hastings and Wysham 2010).

Acknowledgements We wish to thank Dr. John Burke for very helpful discussions and assistance in using AUTO for calculating the bifurcation diagram in Fig. 7. Part of this work was funded by the Department of Energy at Los Alamos National Laboratory under contract DE-AC52-06NA25396 and the DOE Office of Science Advanced Scientific Computing Research (ASCR) program in Applied Mathematics. The research leading to these results has received funding from the European Union Seventh Framework Programme (FP7/2007-2013) under grant number [293825].

Open Access This article is distributed under the terms of the Creative Commons Attribution Noncommercial License which permits any noncommercial use, distribution, and reproduction in any medium, provided the original author(s) and source are credited.

\section{References}

Avitabile D, Lloyd DJB, Burke J, Knobloch E, Sandstede B (2010) To snake or not to snake in the planar SwiftHohenberg equation. SIAM J Appl Dyn Sys 9:704-733. doi:10.1137/100782747

Bailey RM (2010) Spatial and temporal signatures of fragility and threshold proximity in modelled semi-arid vegetation. Proc R Soc B 278:1064-1071

Bergeon A, Burke J, Knobloch E, Mercader I (2008) Eckhaus instability and homoclinic snaking. Phys Rev E 78(4):046,201. doi:10.1103/PhysRevE.78.046201

Borgogno F, D'Odorico P, Laio F, Laio F (2009) Mathematical models of vegetation pattern formation in ecohydrology. Rev Geophys 47:RG1005. doi:10.1029/2007RG000256

Breshears D, Cobb N, Rich P, Price K, Allen C, Balice R, Romme W, Kastens J, Floyd M, Belnap J, et al. (2005) Regional vegetation die-off in response to global-change-type drought. Proc Natl Acad Sci USA 102(42):15,144-15,148

Burke J, Knobloch E (2006) Localized states in the generalized Swift-Hohenberg equation. Phys Rev E 73:056,211

Burke J, Knobloch E (2007) Snakes and ladders: localized states in the Swift-Hohenberg equation. Phys Lett A 360(6):681688. doi:10.1016/j.physleta.2006.08.072

Carpenter SR, Brock WA (2006) Rising variance: a leading indicator of ecological transition. Ecol Lett 9:308-315

Carpenter SR, Brock WA (2010) Early warnings of regime shifts in spatial dynamics using the discrete fourier transform. Ecosphere 1:art10. doi:10.1890/ES10-00016.1 
Chapman S, Kozyreff G (2009) Exponential asymptotics of localised patterns and snaking bifurcation diagrams. Physica D 238(3):319-354. doi:10.1016/j.physd.2008.10.005

Chisholm RA, Filotas E (2009) Critical slowing down as an indicator of transitions in two-species models. J Theor Biol 257:142-149

Cross M, Greenside H (2009) Pattern formation and dynamics in nonequilibrium systems. Cambridge University Press, Cambridge

Dakos V, Scheffer M, van Nes EH, Brovkin V, Petoukhov V, Held H (2008) Slowing down as an early warning signal for abrupt climate change. Proc Natl Acad Sci U S A 105(38):14,308-14,312. doi:10.1073/pnas.0802430105, http://www.pnas.org/content/105/38/14308.full.pdf+html

Dakos V, van Nes EH, Donangelo R, Fort H, Scheffer M (2010) Spatial correlation as leading indicator of catastrophic shifts. Theor Ecol 3:163-174

Dakos V, Kéfi S, Rietkerk M, van Nes E, Scheffer M (2011) Slowing down in spatially patterned ecosystems at the brink of collapse. Am Nat 177(5):E153-66. doi:10.1086/659945

Dawes JHP (2008) Localized pattern formation with a large-scale mode: slanted snaking. SIAM J Appl Dyn Sys 7:186-206

Donangelo R, Fort H, Dakos V, Scheffer M, van Nes EH (2010) Early warnings in ecosystems: comparison between spatial and temporal indicators. Int J Bifurc Chaos Appl Sci Eng 20(8):315-321. doi:10.1142/S0218127410025764

Duraiappah AK, Naeem S (2005) Ecosystems and human wellbeing: biodiversity synthesis. Tech. Rep. of the Millennium Ecosystem Assessment, World Resources Institute, Washington, DC. www.millenniumassessment.org/documents/ document.354.aspx.pdf

Fife PC (1988) Dynamics of internal layers and diffusive interfaces, CCBMS-NSF Regional Conf. Series in Applied Math, vol 53. Society for Industrial and Applied Mathematics, Philadelphia

Firth WJ, Columbo L, Scroggie AJ (2007) Proposed resolution of theory-experiment discrepancy in homoclinic snaking. Phys Rev Lett 99(10):104,503. doi:10.1103/PhysRevLett.99. 104503

Gilad E, von Hardenberg J, Provenzale A, Shachak M, Meron E (2004) Ecosystem engineers: From pattern formation to habitat creation. Phys Rev Lett 93(098105)

Gilad E, von Hardenberg J, Provenzale A, Shachak M, Meron E (2007) A mathematical model for plants as ecosystem engineers. J Theor Biol 244:680

Guttal V, Jayaprakash C (2007) Impact of noise on bistable ecological systems. Ecol Model 201(3-4):420-428. doi:10.1016/ j.ecolmodel.2006.10.005

Guttal V, Jayaprakash C (2008) Changing skewness: an early warning signal of regime shifts in ecosystems. Ecol Lett 11:450-460

Guttal V, Jayaprakash C (2009) Spatial variance and spatial skewness: leading indicators of regime shifts in spatial ecological systems. Theor Ecol 2:3-12

Hagberg A, Meron E (1994a) Complex patterns in reactiondiffusion systems: a tale of two front instabilities. Chaos 4(3):477-484

Hagberg A, Meron E (1994b) From labyrinthine patterns to spiral turbulence. Phys Rev Lett 72(15):2494-2497

Hagberg A, Meron E (1994c) Pattern formation in non-gradient reaction-diffusion systems: the effects of front bifurcations. Nonlinearity 7:805-835

Hagberg A, Meron E (1998) Order parameter equations for front transitions: nonuniformly curved fronts. Physica D 123:460

Hastings A, Wysham DB (2010) Regime shifts in ecological systems can occur with no warning. Ecol Lett 13(464-472)
HilleRisLambers R, Rietkerk M, Van den Bosch F, Prins H, de Kroon H (2001) Vegetation pattern formation in semiarid grazing systems. Ecology 82:50-61

Holling C (1973) Resilience and stability of ecological systems. Ann Rev Ecolog Syst 4:1-23

Kéfi S, Rietkerk M, Alados CL, Pueyo Y, Papanastasis VP, ElAich A, de Ruiter PC (2007) Spatial vegetation patterns and imminent desertification in Mediterranean arid ecosystems. Nature 449:213-217

Kéfi S, Rietkerk M, Roy M, Franc A, de Ruiter PC, Pascual M (2011) Robust scaling in ecosystems and the meltdown of patch size distributions before extinction. Ecol Lett 14(1):29-35

Kletter AY, von Hardenberg J, Meron E (2012) Ostwald ripening in dryland vegetation. Comm Pure Appl Anal 11:261273

Knobloch E (2008) Spatially localized structures in dissipative systems: open problems. Nonlinearity 21(4):T45

Kozyreff G, Chapman SJ (2006) Asymptotics of large bound states of localized structures. Phys Rev Lett 97(4):044,502. doi:10.1103/PhysRevLett.97.044502

Lejeune O, Couteron P, Lefever R (1999) Short range cooperativity competing with long range inhibition explains vegetation patterns. Acta Oecol 20(3):171-183

Lejeune O, Tlidi M, Lefever R (2004) Vegetation spots and stripes: dissipative structures in arid landscapes. Int J Quant Chem 98:261-271

Lewontin RC (1969) The meaning of stability. Brookhaven Symp Biol 22:13-24

Lloyd DJB, Sandstede B, Avitabile D, Champneys AR (2008) Localized hexagon patterns of the planar SwiftHohenberg equation. SIAM J Appl Dyn Sys 7:1049-1100. doi:10.1137/070707622

Loreau $\mathrm{M}$, Naeem S, Inchausti $\mathrm{P}$, Bengtsson $\mathrm{J}$, Grime $\mathrm{J}$, Hector A, Hooper D, Huston M, Raffaelli D, B S, Tilman D, Wardle D (2001) Biodiversity and ecosystem functioning: current knowledge and future challenges. Science 294: 804-808

Manor A, Shnerb NM (2008) Facilitation, competition, and vegetation patchiness: from scale free distribution to patterns. J Theor Bio 253(4):838-842

May RM (1977) Thresholds and breakpoints in ecosystems with a multiplicity of stable states. Nature 296:471-477. doi:10.1038/269471a0

McCalla S, Sandstede B (2010) Snaking of radial solutions of the multi-dimensional Swift-Hohenberg equation: A numerical study. Physica D 239(16):1581-1592. doi:10.1016/j.physd. 2010.04.004

Meerson B, Sasorov PV (1996) Domain stability, competition, growth, and selection in globally constrained bistable systems. Phys Rev E 53(4):3491-3494. doi:10.1103/PhysRevE. 53.3491

Meron E (2011a) Modeling dryland landscapes. Math Model Nat Phenom 6(01):163-187. doi:10.1051/mmnp/20116109

Meron E (2011b) Pattern-formation approach to modelling spatially extended ecosystems. Ecol Model. doi:10.1016/ j.ecolmodel.2011.05.035

Meron E, Yizhaq H, Gilad E (2007) Localized structures in dryland vegetation: forms and functions. Chaos 17 (037109)

Mueller RC, Scudder CM, Porter ME, III RTT, Gehring CA, Whitham TG (2005) Differential tree mortality in response to severe drought: Evidence for long-term vegetation shifts. J Ecol 93(6):1085-1093

Pismen LM (2006) Patterns and interfaces in dissipative dynamics. Springer, New York 
Pomeau Y (1986) Front motion, metastability and subcritical bifurcations in hydrodynamics. Physica D 23:3-11

Rietkerk M, van de Koppel J (2008) Regular pattern formation in real ecosystems. Trends Ecol Evol 23(3):169175

Rietkerk M, Boerlijst M, van Langevelde F, HilleRisLambers R, van de Koppel J, Kumar L, Prins H, De Roos A (2002) Self-organization of vegetation in arid ecosystems. Am Nat 160:524-530

Rietkerk M, Dekker SC, de Ruiter PC, van de Koppel J (2004) Self-organized patchiness and catastrophic shifts in ecosystems. Science 305:1926-1929

Scanlon TM, Caylor KK, Levin SA, Rodriguez-Iturbe I (2007) Positive feedbacks promote power-law clustering of Kalahari vegetation. Nature 449:209-212

Scheffer M, Carpenter SR (2003) Catastrophic regime shifts in ecosystems: linking theory to observation. Trends Ecol Evol 18:648-656

Scheffer M, Carpenter S, Foley J, Folke C, Walkerk B (2001) Catastrophic shifts in ecosystems. Nature 413:591596
Scheffer M, Bascompte J, Brock WA, Brovkin V, Carpenter SR, Dakos V, Held H, van Nes EH, Rietkerk M, Sugihara G (2009) Early-warning signals for critical transitions. Nature 461:387-393

Sherratt JA (2005) An analysis of vegetation stripe formation in semi-arid landscapes. J Math Biol 51:183-197

Strogatz SH (1994) Nonlinear dynamics and chaos: with applications to physics, biology, chemistry and engineering. Perseus Books, Cambridge

Tlidi M, Lefever R, Vladimirov A (2008) On vegetation clustering, localized bare soil spots and fairy circles. Lect Notes Phys 751:381-402

von Hardenberg J, Meron E, Shachak M, Zarmi Y (2001) Diversity of vegetation patterns and desertification. Phys Rev Lett 89(198101)

von Hardenberg J, Kletter AY, Yizhaq H, Nathan J, Meron E (2010) Periodic versus scale-free patterns in dryland vegetation. Proc R Soc B 277:1771-1776

Yachi S, Loreau M (1999) Biodiversity and ecosystem productivity in a fluctuating environment: the insurance hypothesis. Proc Natl Acad Sci U S A 96:1463-1468 